\title{
Achados de imagem na doença de Tay-Sachs: um relato de caso
}

\section{Neuroimaging in Tay-Sachs disease: a case report}

\author{
Suyane Benevides Franco ${ }^{1}$. Pedro Henrique Sales Pontes ${ }^{1}$. Elaine Fernandes Andrade Teixeira ${ }^{1}$. Norma
} Martins de Menezes Morais². Daniel Aguiar Dias³.

1 Médico residente do Programa de Radiologia e Diagnóstico por Imagem, Universidade Federal do Ceará (UFC), Fortaleza, Ceará, Brasil. 2 Mestre em Saúde da Criança e do Adolescente, Médica Neuropediatra, Hospital Universitário Walter Cantídio (HUWC), Fortaleza, Ceará, Brasil. 3 Médico Radiologista, Universidade Federal do Ceará (UFC), Hospital Universitário Walter Cantídio (HUWC), Fortaleza, Ceará, Brasil.

\section{RESUMO}

Objetivos: Revisar os achados de imagem característicos na doença de Tay-Sachs, um distúrbio autossômico recessivo raro. Metodologia: Relato de caso de um paciente do sexo masculino, de 1 ano e 6 meses de idade, com atraso no desenvolvimento neuropsicomotor e convulsões, que realizou exames de imagem no nosso serviço. Resultados: A tomografia computadorizada de crânio revelou, como principal achado, hiperdensidade espontânea nos tálamos, além de hipodensidade simétrica e difusa na substância branca supratentorial. A ressonância magnética de crânio mostrou alteração de sinal na substância branca nas imagens ponderadas em T2, com hipersinal na substância branca periventricular, profunda e subcortical, núcleos da base, cápsulas internas, externas e extremas, com preservação relativa do corpo caloso. Além disso, foi observado hipersinal espontâneo em T1 nos tálamos. Os corpos estriados exibiam volumes aumentados, com múltiplos focos puntiformes hipotensos em T2 (aparência manchada/ salpicada). A espectroscopia de prótons revelou diminuição da razão N-acetil aspartato (NAA)/creatina (Cr) e aumento da proporção de mioinositol (mI)/Cr. A fundoscopia demonstrou manchas vermelhas maculares (cherry red spots). Conclusões: A doença de Tay-Sachs está associada a alterações de imagem fortemente sugestivas, podendo ser corroborado pela espectroscopia, devendo ser reconhecidas pelo médico radiologista para adequado diagnóstico diferencial das doenças neurológicas hipomielinizantes da infância.

Palavras-chave: Gangliosidoses GM2. Ressonância magnética. Tomografia computadorizada. Espectroscopia.

\section{ABSTRACT}

Objectives: To review the characteristic imaging findings in Tay-Sachs disease, a rare autosomal recessive disorder. Methodology: Case report of 18-month-old male patient with delayed neuropsychomotor development and seizures that underwent imaging evaluation at our service. Results: Computed tomography of the skull revealed spontaneous hyperdensities in the thalami, as well as symmetrical and diffuse hypodensity in the supratentorial white matter. Magnetic resonance imaging of the brain showed white matter T2 signal alterations, with hyperintensities in the deep periventricular and subcortical white matter, basal ganglia and internal and external capsules, with relative preservation of the corpus callosum. In addition, spontaneous T1 hyperintensity was observed in the thalamus. Striated bodies exhibited increased volumes, with multiple hypointense punctate foci in T2 (spotted/ speckled appearance). Proton spectroscopy revealed a decrease in the $\mathrm{N}$-acetyl aspartate (NAA)/creatine (Cr) ratio and an increase in the myo-inositol $(\mathrm{mI}) /(\mathrm{Cr})$ ratio. Fundoscopy showed red spots ("cherry red spots"). Conclusions: Tay-Sachs disease is associated with strongly suggestive imaging alterations, which can be corroborated by spectroscopy and should be recognized by the radiologist for an adequate differential diagnosis of childhood hypomielinizing neurological diseases.

Keywords: GM2 gangliosidosis. Magnetic resonance imaging. Computed tomography. Spectroscopy.

Autor correspondente: Suyane Benevides Franco, Av Historiador Raimundo Girão, 860, Meireles, Fortaleza, Ceará. CEP: 60165-050. Telefone: +55 85 98848-1986. E-mail: suyanebenevides@hotmail.com

Conflito de interesses: Não há qualquer conflito de interesses por parte de qualquer um dos autores.

Recebido em: 04 Jan 2018; Revisado em: 17 Mai 2018; Aceito em: 17 Mai 2018. 


\section{INTRODUÇÃO}

A gangliosidose GM2 é um distúrbio autossômico recessivo, que consiste em uma alteração lisossômica, bioquimicamente caracterizada por uma incapacidade de hidrolisar o gangliosídio GM2, que se acumula particularmente nas células neuronais. ${ }^{1}$ A deficiência das isoenzimas hexosaminidase A e hexosaminidase B produzidas por diferentes mutações do gene que codifica a subunidade $\beta$ é a causa da variante da gangliosidose GM2, conhecida como doença de Sandhoff. Por sua vez, a deficiência isolada da isoenzima hexosaminidase A, produzida por diferentes mutações do gene que codifica a subunidade $\alpha$, é conhecida como doença de Tay-Sachs. ${ }^{2,3}$

Tanto na doença de Tay-Sachs, como na doença de Sandhoff há acúmulo dos gangliosídios GM2 no sistema nervoso central, provocando comprometimento da função neuronal. $\mathrm{Na}$ doença de Sandhoff, entretanto, está associada a hepatoesplenomegalia. ${ }^{4}$ Este artigo apresenta os achados de tomografia computadorizada (TC), de ressonância magnética (RM) e de espectroscopia de RM em uma criança com doença de Tay-Sachs.

\section{RELATO DE CASO}

Um paciente do sexo masculino, de 1 ano e 6 meses de idade, nascido no Brasil, filho de pais consanguíneos com relato de descendência judaica, com macrocefalia, evoluindo com convulsões refratárias, atraso no desenvolvimento neuropsicomotor, perda de marcos adquiridos, além de tetraparesia espástica.

A TC de crânio revelou hipodensidade simétrica e difusa na substância branca supratentorial bilateral, com tálamos espontaneamente hiperdensos (Figura 1). Além disso, observou-se também leve atrofia cortical difusa. Entretanto, as estruturas infratentoriais estavam normais ao método. $\mathrm{O}$ paciente foi submetido a uma ultrassonografia do abdome em que não foram observadas alterações.

Figura 1. Corte axial de tomografia computadorizada do crânio. Observa-se hiperdensidade espontânea dos tálamos e hipodensidade simétrica e difusa na substância branca supratentorial bilateral. Notar ainda leve atrofia cortical difusa para a faixa etária do paciente.

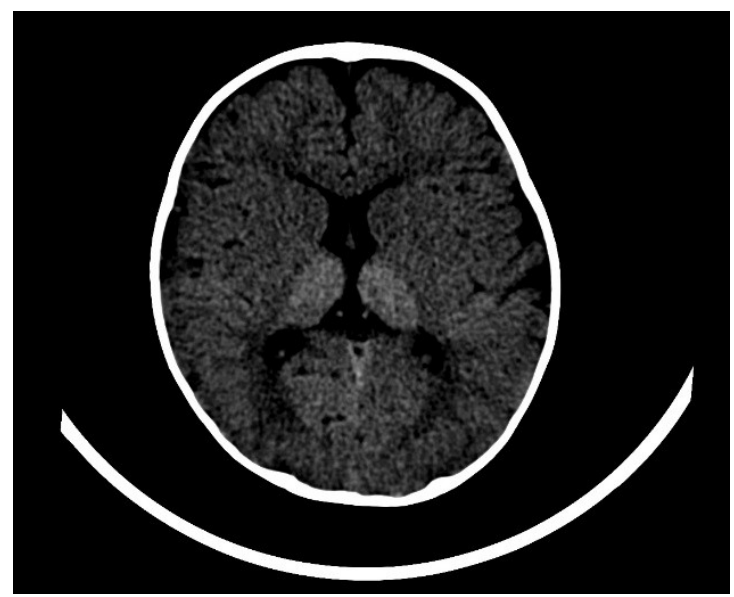

A RM de crânio mostrou aumento da intensidade do sinal na substância branca periventricular, profunda e subcortical, corpus striatum, cápsulas internas/externas e nas lâminas medulares interno, medial e lateral, com preservação do corpo caloso (Figura 2). Além disso, foi observado hipersinal espontâneo em T1 nos tálamos (Figura 3) e núcleos caudados/ lentiforme com volumes aumentados, exibindo múltiplos focos puntiformes hipotensos em T2 (aparência manchada/ salpicada) (Figura 4). Após a administração do meio de contraste paramagnético não foram observados realces anômalos.

Figura 2. Corte axial de ressonância magnética do crânio na sequência FLAIR/T2 mostrando hipersinal na substância branca periventricular, profunda e subcortical, corpus striatum, cápsulas internas/externas e nas lâminas medulares interno, medial e lateral, com preservação do corpo caloso.

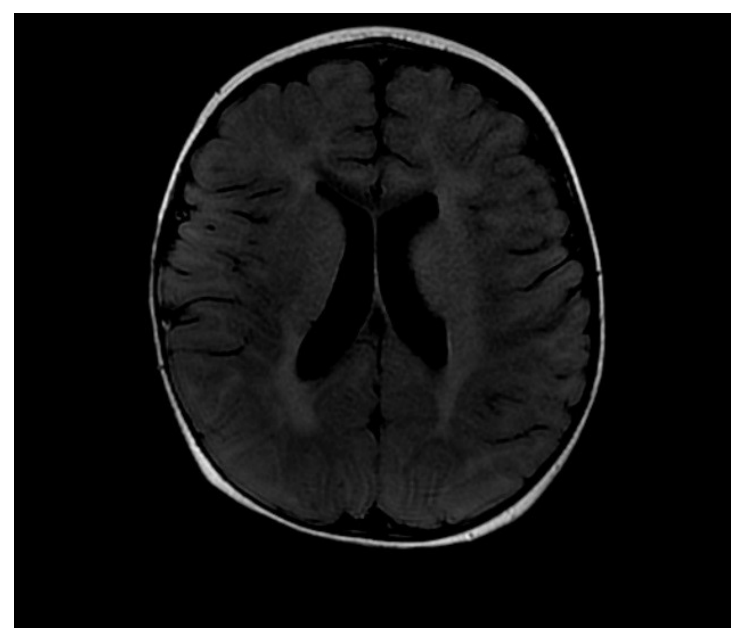

Figura 3. Corte axial de ressonância magnética do crânio na sequência T1 mostrando hipersinal nos tálamos.

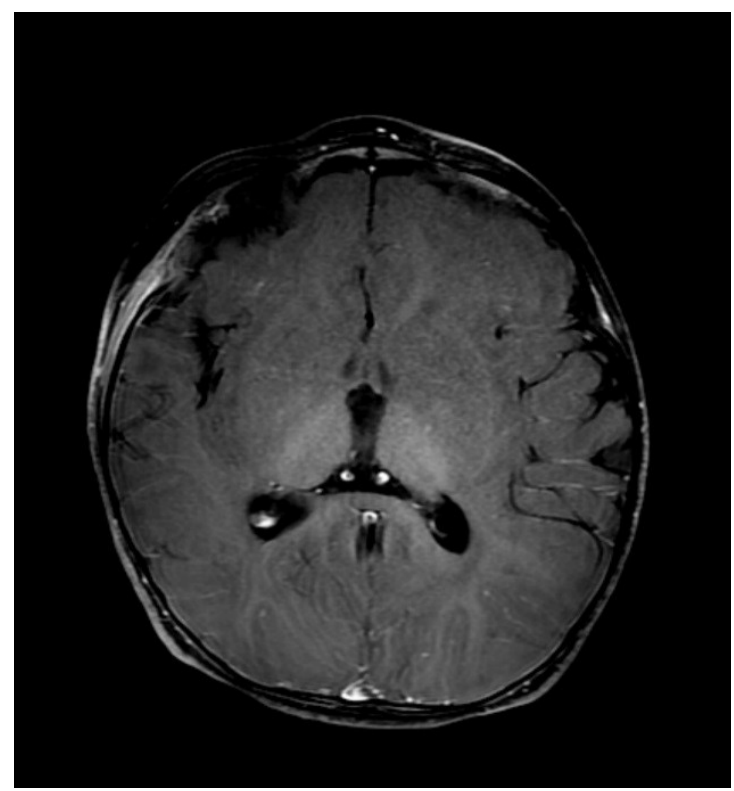


Figura 4. Corte axial de ressonância magnética do crânio na sequência FLAIR/T2 mostrando núcleos caudados/lentiforme com volumes aumentados, exibindo múltiplos focos puntiformes hipotensos em T2 (aparência manchada/salpicada).

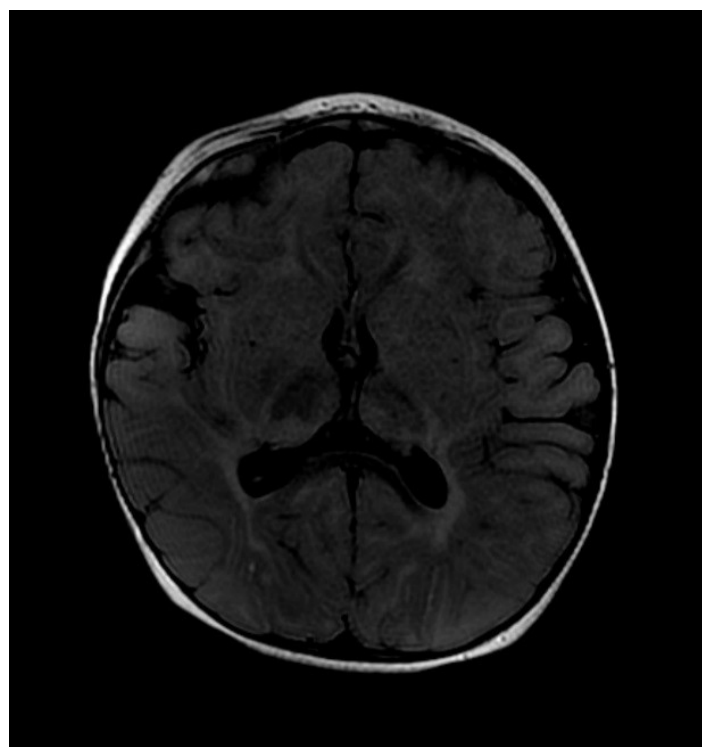

$\mathrm{Na}$ espectroscopia de prótons (Figura 5), os valores integrais dos picos de metabólitos e suas relações foram obtidos em duas regiões de interesse (ROI), utilizando o voxel único com tempo de eco (TE) de $35 \mathrm{mseg}$ e de 144 mseg nos núcleos da base. Os espectros de prótons revelaram diminuição da razão $\mathrm{N}$-acetil aspartato $(\mathrm{NAA}) /$ creatina $(\mathrm{Cr})$, aumento da proporção de mioinositol (mI)/Cr e de colina normal (Cho)/ $\mathrm{Cr}$ em comparação com a espectroscopia normal para idade. Foram observados também pequenos picos entre 0,9 e 1,6 ppm.

Figura 5. Na espectroscopia de prótons, os valores integrais dos picos de metabólitos e suas relações foram obtidos em duas regiões de interesse (ROI), utilizando o voxel único com tempo de eco (TE) de $35 \mathrm{mseg}$ (Figura 5.a) e de $144 \mathrm{mseg}$ (Figura 5.b). Os espectros de prótons revelaram diminuição da razão (NAA)/Cr, aumento da proporção de $\mathrm{mI} / \mathrm{Cr}$ em comparação com a espectroscopia normal para idade. Foi observado também, pequenos picos entre 0,9 e 1,6 ppm (seta).

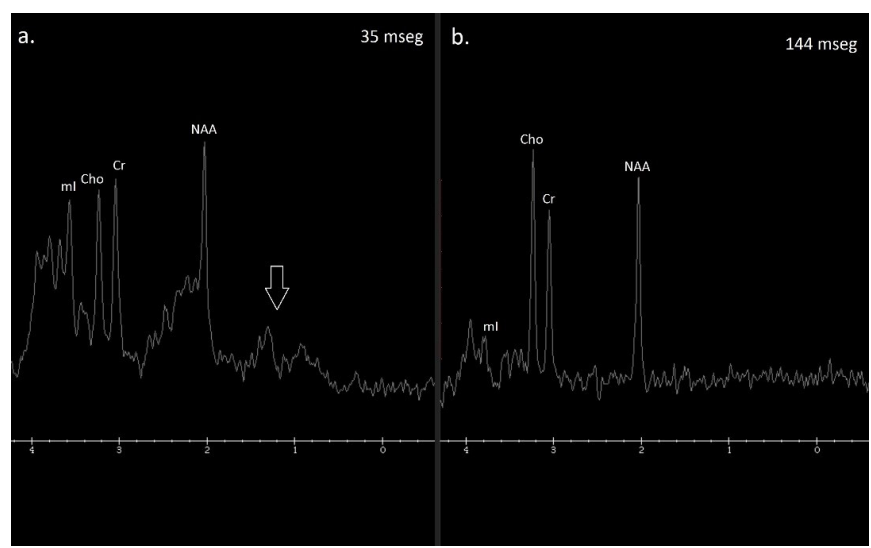

A fundoscopia demonstrou manchas vermelhas na área macular bilateralmente (cherry red spots) (Figura 6).

Foi realizada a dosagem total plasmática da enzima hexosaminidase A no paciente, demonstrando marcada diminuição da atividade enzimática e confirmando o diagnóstico de Doença de Tay-Sachs.

Figura 6. A fundoscopia demonstrou manchas vermelhas (setas) na área macular dos dois globos oculares (cherry red spots).
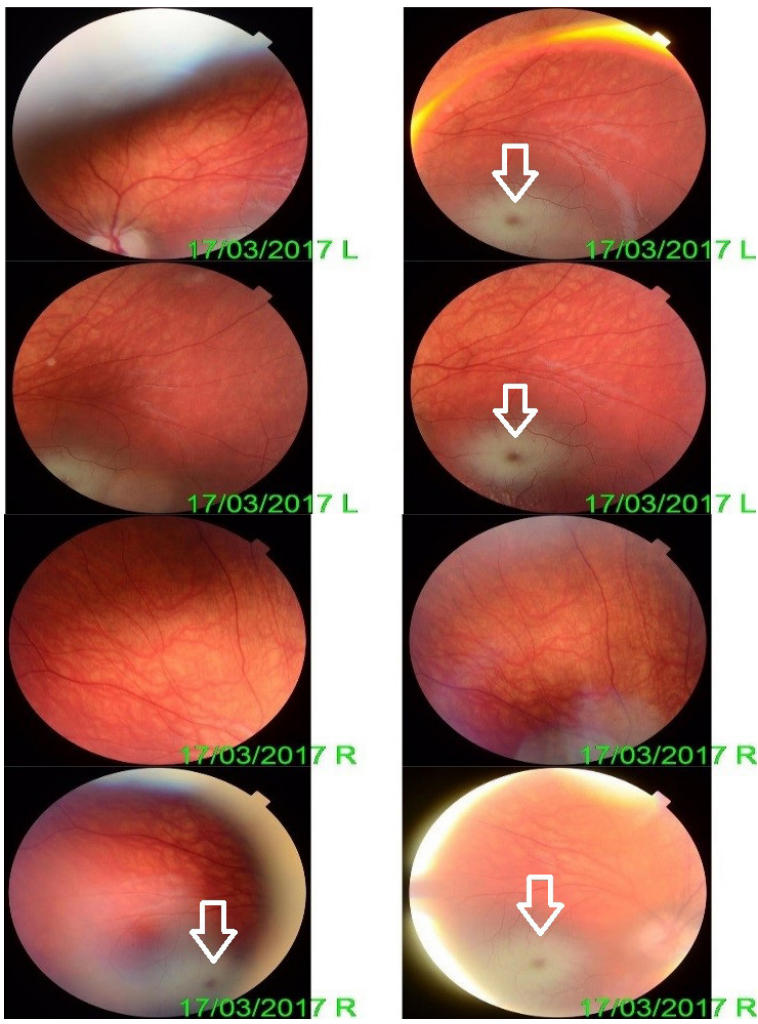

\section{DISCUSSÃO}

A incidência da doença de Tay-Sachs varia entre diferentes grupos étnicos, sendo 0,54 por 100.000 nascidos vivos nos Estados Unidos. A prevalência na população judaica Ashkenazi é bem mais elevada, sendo estimada de 1 a cada 25 indivíduos.

Os indivíduos portadores das mutações de Tay Sachs mostram um nível reduzido da enzima hexosaminidase A que é essencial para quebrar substâncias gordurosas no tecido neural. Como resultado, essas moléculas lipídicas, os gangliosídeos, se acumulam no sistema nervoso central e gradualmente destroem os neurônios. Os gangliósidos GM2 são compostos por uma esfingosina acetilada com uma cadeia de oligossacarídeos anexada. A deficiência da subunidade $\alpha$ da enzima $\beta$-N-acetil galactosaminidase, que hidrolisa a $\mathrm{N}$-acetilgalactosamina terminal dos gangliósidos GM2 nos lisossomos, resulta no acúmulo de gangliósidos GM2 nos lisossomos e citoplasma neuronal. A deposição dos 
gangliósidos GM2 nos neurônios leva a distorção estrutural e consequente redução do número de neurônios com a progressão da doença. ${ }^{5} \mathrm{O}$ NAA é um marcador de integridade neuroaxonal na espectroscopia de RM do protão e uma diminuição da NAA está associada à função prejudicada dos neurônios e/ou lesão axonal.

Na doença de Tay-Sachs, a desmielinização ativa e grave na substância branca central que poupa a cápsula interna e o corpo caloso acompanha a morte neuronal. No estágio final do processo da doença, a desmielinização pode ser generalizada na substância branca cerebral. O pico de Cho na espectroscopia de prótons é conhecido por conter contribuições de fosforilcolina e glicerofosforilcolina, que são os precursores da síntese da membrana celular e o produto da ruptura da membrana celular. Assim, um aumento de pico Cho é um indicador de desmielinização ativa. A desmielinização foi relatada como parte dos achados neuropatológicos em pacientes com doença de Tay-Sachs. Os pequenos picos de 0,9-1,6 ppm observados no caso relatado e em outros casos da literatura, podem resultar dos prótons de metil e metileno de cadeias de ácidos de gordura criados pelos produtos de degradação da desmielinização. ${ }^{6}$

O principal achado doença de Tay-Sachs na imagem de TC é a hiperdensidade espontânea nos tálamos. Já hiperintensidade nas imagens ponderadas em T2 nos gânglios basais, o tálamo e a substância branca cerebral, poupando a cápsula interna, são os principais achados de RM. As lesões na doença precoce de Tay-Sachs começam nos gânglios basais e na substância branca periventricular. À medida que a doença progride, as imagens de RM demonstram disseminação subcortical das lesões de substância branca e atrofia cerebral., ${ }^{7,9}$

\section{REFERÊNCIAS}

1. O'Brien JS. The gangliosidoses. In: Stanbury JB, Wijngaarden JB, Fredericksen DS (eds). The metabolic basis of inherited disease. New York: McGraw-Hill; 1983. p. 5945-967.

2. Haghighi A, Masri A, Kornreich R, Desnick RJ. Tay-Sachs disease in an Arab family due to c78G $>$ A HEXA nonsense mutation encoding a pW26X early truncation enzyme peptide. Mol Genet Metab. 2011;104(4):700-2.

3. Rosengren B, Månsson JE, Svennerholm L. Composition of gangliosides and neutral glycosphingolipids of brain in classical tay-sachs and sandhoff disease: more lyso-GM2 in sandhoff disease? Journal of Neurochemistry. 1987;49:834-40.

4. Karimzadeh P, Jafari N, Nejad Biglari H, Jabbeh Dari S, Ahmad Abadi F, Alaee MR, et al. GM2-gangliosidosis (sandhoff and tay sachs disease): diagnosis and neuroimaging findings (an Iranian pediatric case series). Iran J Child Neurol. 2014;8:55-60.
Além dos ensaios enzimáticos da atividade da hexosaminidase A, o diagnóstico também pode ser feito através de testes genéticos usando reação em cadeia da polimerase. Além da atividade de ensaio enzimático e triagem genética, exames oculares também podem ajudar no diagnóstico de Tay Sachs. Conforme mencionado no relato do caso, os indivíduos afetados apresentam um achado característico cherry red spots no olho devido ao deslocamento do nervo óptico.

\section{CONCLUSÃO}

Existem achados de imagem caracteristicamente encontrados na doença de Tay-Sachs. Os principais achados na TC de crânio são a hiperdensidade espontânea dos tálamos e hipodensidade da substância branca, podendo ou não haver algum grau de atrofia associado a depender do estágio da doença. Na RM, observa-se alteração de sinal na substância branca nas imagens ponderadas em T2, com preservação do corpo caloso, hipersinal espontâneo em T1 dos tálamos, além de hipersinal nos núcleos caudados/lentiforme com volumes aumentados, exibindo múltiplos focos puntiformes hipotensos em T2 (aparência manchada/salpicada). Na espectroscopia de prótons de RM, foi observado diminuição da razão (NAA)/ $\mathrm{Cr}$ e aumento da proporção de $\mathrm{mI} / \mathrm{Cr}$. Há ainda o achado característico da fundoscopia, que são manchas vermelhas na área macular bilateralmente (cherry red spots).

Os achados acima descritos, juntamente com a clínica de alterações neurológicas, permitiram suspeitar fortemente do diagnóstico de doença de Tay-Sachs. O caso apresentado foi confirmado através da dosagem total plasmática da enzima hexosaminidase $\mathrm{A}$, demonstrando marcada diminuição da atividade enzimática.

5. Knaap, MS, Valk J. Magnetic resonance of myelin, myelination and myelin disorders. New York: Springer; 1995.

6. Aydin K, Bakir B, Tatli B, Terzibasioglu E, Ozmen M. Proton MR spectroscopy in three children with Tay-Sachs disease. Pediatr Radiol. 2005;35(11):1081-5.

7. Fukumizu M, Yoshikawa H, Takashima S, Sakuragawa N, Kurokawa T. Tay-Sachs disease: progression of changes on neuroimaging in four cases. Neuroradiology. 1992;34(6):483-6.

8. Mugikura S, Takahashi S, Higano S, Kurihara N, Kon K, Sakamoto K. MR Findings in Tay-Sachs disease. J Comput Assist Tomogr. 1996;20(4):551-5.

9. Mittal P, Gupta R, Garg P, Mittal A, Kaur H, Gupta S. CT and MRI findings in a case of infantile form of GM2 gangliosidosis: Tay-Sachs disease. Neurol India. 2016;64:1372-3.

\section{Como citar:}

Franco SB, Pontes PH, Teixeira EF, Morais NM, Dias DA. Achados de imagem na doença de Tay-Sachs: um relato de caso. Rev Med UFC. 2018 out-dez;58(4):57-60. 